\title{
Regularity results for eikonal-type equations with nonsmooth coefficients
}

\author{
Piermarco Cannarsa and Pierre Cardaliaguet
}

\begin{abstract}
Solutions of the Hamilton-Jacobi equation $H(x,-D u(x))=1$, where $H(\cdot, p)$ is Hölder continuous and the level-sets $\{H(x, \cdot) \leq 1\}$ are convex and satisfy positive lower and upper curvature bounds, are shown to be locally semiconcave with a power-like modulus. An essential step of the proof is the $\mathcal{C}^{1, \alpha}$-regularity of the extremal trajectories associated with the multifunction generated by $D_{p} H$.
\end{abstract}

Mathematics Subject Classification (2000). 49L25, 34A60, 26B25, 49N60.

Keywords. Viscosity solutions, Semiconcave functions, Differential inclusions, Extremal trajectories.

\section{Introduction}

The importance of semiconcavity for the study of Hamilton-Jacobi equations and optimal control problems is by now widely acknowledged. Indeed, such a qualitative property ensures the upper semicontinuity and quasi-monotonicity of the superdifferential of solutions. Moreover, it can be used to derive upper bounds for the set where solutions fail to be differentiable, providing at the same time criteria for the propagation of singularities. Finally, it leads to stronger optimality conditions than the ones holding for a continuous (or Lipschitz continuous) function, see, for instance, [3] and the references therein.

Typically, a real-valued function $u$ is semiconcave on the convex set $D \subset$ $\mathbb{R}^{N}$ if there exists a modulus (i.e., nondecreasing upper semicontinuous function, vanishing at 0$) \omega:[0, \infty) \rightarrow[0, \infty)$ such that

$$
u(\lambda x+(1-\lambda) y) \geq \lambda u(x)+(1-\lambda) u(y)-C \lambda(1-\lambda)|x-y| \omega(|x-y|)
$$

for all $x, y \in D$ and $\lambda \in[0,1]$.

Semiconcavity results with a linear modulus hold for viscosity solutions of Hamilton-Jacobi equations with convex Hamiltonians which are sufficiently smooth with respect to the space variables, as well as for value functions of 
optimal control problems with smooth dynamics and running cost (see, e.g., $[2,6,7]$; see also [3]). Known generalizations allow for Lipschitz continuous dependance with respect to space, provided the Hamiltonian is strictly convex and superlinear in the gradient variables (see [4,9]).

In this paper we shall study the Dirichlet problem

$$
\begin{cases}H(x,-D u(x))=1 & \text { in } \Omega \\ u(x)=0 & \text { on } \partial \Omega\end{cases}
$$

where $\Omega$ is an open subset of $\mathbb{R}^{N}, H(x, \cdot)$ is convex and positively homogeneous of degree 1 , and $H(\cdot, p)$ is just Hölder continuous. Consequently, (1) fits none of the aforementioned settings. Nevertheless, our main result-Theorem 5.1 below - guarantees that the solution $u$ of (1) is locally semiconcave in $\Omega$ with the power-like modulus $\omega(t)=C t^{\theta}$, for some $\theta>0$ depending on $H$. Our key assumption is that the curvature of the level set $\{H(x, \cdot) \leq 1\}$ is bounded above and below by positive constants uniformly with respect to the space variable $x$ (see assumption (5), as well as the geometric property (11)).

The method of proof relies on the representation of $u(x)$ as the minimum time needed to reach $\partial \Omega$ along a trajectory of the differential inclusion

$$
\left\{\begin{array}{l}
x^{\prime}(t) \in F(x(t)) t \geq 0 \quad \text { a.e. } \\
x(0)=x
\end{array}\right.
$$

where

$$
F(x)=\operatorname{co}\left\{D_{p} H(x, p): p \in \mathbb{R}^{N} \backslash\{0\}\right\} \quad \forall x \in \mathbb{R}^{N} .
$$

An essential step of the analysis is the $\mathcal{C}^{1, \alpha}$-regularity of the extremal trajectories of (2), see Theorem 4.1. Related results hold for geodesics on manifolds with Hölder continuous Riemannian or Finsler metrics, as explained in [8]. The problem of interest to our paper, however, does not fit this framework because we do not assume $H$ to be symmetric with respect to $p$ (see Example 2.1). For time-dependent and isotropic Hamiltonians $(H=a(t, x)|p|)$, the above regularity property has also been observed in [10] for $N=2$, and [5] for general $N$. However, the unexpected connection between Theorem 4.1 and the semiconcavity of the solution of (1) is, to our best knowledge, entirely new.

The outline is the following: Sect. 2 contains our basic notations and assumptions. In Sect. 3, we describe the main technical tools we borrow from convex analysis, making this paper essentially self-contained. In Sect. 4, we prove our regularity results for extremal trajectories, which is applied, in Sect. 5, to derive the semiconcavity estimate for the solution of (1).

\section{Notation and assumptions}

Let $N$ be a positive integer. Denote by $\langle\cdot, \cdot\rangle$ and $|\cdot|$ the Euclidean scalar product and norm in $\mathbb{R}^{N}$, respectively, and set

$$
B=\left\{x \in \mathbb{R}^{N}:|x| \leq 1\right\} .
$$

More generally, for all $x \in \mathbb{R}^{N}$ and $\rho>0, B(x, \rho)$ stands for the closed ball of radius $\rho$ centered at $x$, that is, $B(x, \rho)=x+\rho B$. 
Let $H: \mathbb{R}^{N} \times \mathbb{R}^{N} \rightarrow \mathbb{R}$ be a continuous function satisfying the following assumptions for some positive constants $C_{0}, r, R$, with $r<R$, and $\alpha \in(0,1 / 2)$. Standing Assumptions (SA):

- For all $p \in \mathbb{R}^{N}$, the function $x \mapsto H(x, p)$ is $2 \alpha$-Hölder continuous, and

$$
|H(x, p)-H(y, p)| \leq C_{0}|x-y|^{2 \alpha}|p| \quad \forall x, y \in \mathbb{R}^{N} .
$$

- For all $x \in \mathbb{R}^{N}$, the function $p \mapsto H(x, p)$ is convex on $\mathbb{R}^{N}$, positively homogeneous of degree one, and has linear growth, i.e.,

$$
r|p| \leq H(x, p) \leq R|p| \quad \forall p \in \mathbb{R}^{N}
$$

- For all $x \in \mathbb{R}^{N}$, the function $p \mapsto H(x, p)$ is continuously differentiable on $\mathbb{R}^{N} \backslash\{0\}$, and, for all $p, q \in \mathbb{R}^{N} \backslash\{0\}$,

$$
\begin{aligned}
-\frac{1}{2 r} & \left|D_{p} H(x, q)-D_{p} H(x, p)\right|^{2} \\
& \leq\left\langle D_{p} H(x, q)-D_{p} H(x, p), \frac{p}{|p|}\right\rangle \\
& \leq-\frac{1}{2 R}\left|D_{p} H(x, q)-D_{p} H(x, p)\right|^{2},
\end{aligned}
$$

where $D_{p} H(x, p)$ denotes the gradient of $H$ in the $p$-variables at $(x, p)$.

Our key assumption is (5), which means, roughly speaking, that the curvature of the level set $\{H(x, \cdot) \leq 1\}$ is bounded above and below by positive constants uniformly with respect to the space variable $x$ : see Lemma 3.1 below.

Hereafter, by a universal constant - briefly, a constant-we mean a positive real number that only depends on the parameters $N, \alpha, r, R$, and $C_{0}$ introduced above. Generic constants appearing in computations will be denoted by $C$. A subscript $\left(C_{1}, C_{2}, \ldots\right)$ will be added when necessary for future reference.

The following example illustrates the typical set-up of interest to this paper.

Example 2.1. Let $H(x, p)=\langle g(x), p\rangle+|A(x) p|$, where $g: \mathbb{R}^{N} \rightarrow \mathbb{R}^{N}$ and $A: \mathbb{R}^{N} \rightarrow \mathbb{R}^{N \times N}$ are $2 \alpha$-Hölder continuous on $\mathbb{R}^{N}, A(x)$ is invertible for all $x \in \mathbb{R}^{N}$, and

$$
|g(x)| \leq \frac{C}{2}, \quad|A(x)| \leq C \quad \text { and } \quad\left|A(x)^{-1}\right| \leq C \quad \forall x \in \mathbb{R}^{N}
$$

for some constant $C$. Then $H$ satisfies (SA) for a suitable choice of constants. Indeed (3) and (4) follow directly from the definition of $H$ and (6). Moreover, since $D_{p} H(p)=\frac{A^{*} A p}{|A p|}$ (hereafter we drop $x$-dependence), we have

$$
\left|D_{p} H(q)-D_{p} H(p)\right|^{2}=\left|\frac{A^{*} A q}{|A q|}-\frac{A^{*} A p}{|A p|}\right|^{2} \leq\left\|A^{*}\right\|^{2}\left|\frac{A q}{|A q|}-\frac{A p}{|A p|}\right|^{2} .
$$

Then,

$$
\left|\frac{A q}{|A q|}-\frac{A p}{|A p|}\right|^{2}=2\left[1-\left\langle\frac{A q}{|A q|}, \frac{A p}{|A p|}\right\rangle\right]=2 \frac{|p|}{|A p|}\left\langle\frac{A p}{|A p|}-\frac{A q}{|A q|}, \frac{A p}{|p|}\right\rangle .
$$


Now, from our assumption on $A^{-1}, \frac{|p|}{|A p|}$ is bounded above by some constant $M>0$. Therefore,

$$
\begin{aligned}
\left|D_{p} H(q)-D_{p} H(p)\right|^{2} & \leq 2\left\|A^{*}\right\|^{2} M\left\langle\frac{A^{*} A p}{|A p|}-\frac{A^{*} A q}{|A q|}, \frac{p}{|p|}\right\rangle \\
& =2\left\|A^{*}\right\|^{2} M\left\langle D_{p} H(p)-D_{p} H(q), \frac{p}{|p|}\right\rangle,
\end{aligned}
$$

which gives the first inequality in (5). As for the second one,

$$
\left|D_{p} H(q)-D_{p} H(p)\right|^{2}=\left|\frac{A^{*} A q}{|A q|}-\frac{A^{*} A p}{|A p|}\right|^{2} \geq \frac{1}{\left\|A^{-*}\right\|^{2}}\left|\frac{A q}{|A q|}-\frac{A p}{|A p|}\right|^{2},
$$

where $A^{-*}=\left(A^{-1}\right)^{*}$. Thus,

$$
\left|\frac{A q}{|A q|}-\frac{A p}{|A p|}\right|^{2}=2\left[1-\left\langle\frac{A q}{|A q|}, \frac{A p}{|A p|}\right\rangle\right]=2 \frac{|p|}{|A p|}\left\langle\frac{A p}{|A p|}-\frac{A q}{|A q|}, \frac{A p}{|p|}\right\rangle .
$$

Now, from our assumption on $A^{-1}, \frac{|p|}{|A p|}$ is bounded below by some constant $1 / M>0$. So,

$$
\begin{aligned}
\left|D_{p} H(q)-D_{p} H(p)\right|^{2} & \geq \frac{2}{\left\|A^{-*}\right\|^{2} M}\left\langle\frac{A^{*} A p}{|A p|}-\frac{A^{*} A q}{|A q|}, \frac{p}{|p|}\right\rangle \\
& =\frac{2}{\left\|A^{-*}\right\|^{2} M}\left\langle D_{p} H(p)-D_{p} H(q), \frac{p}{|p|}\right\rangle .
\end{aligned}
$$

Note that, in contrast with the conditions in [8], $H$ is not symmetric with respect to the $p$ variable whenever $g \not \equiv 0$.

\section{Preliminary results}

Let $H: \mathbb{R}^{N} \times \mathbb{R}^{N} \rightarrow \mathbb{R}$ be a continuous function satisfying our Standing Assumptions with fixed constants $\alpha, r, R$, and $C_{0}$.

For all $p \neq 0$, set $f_{p}(x)=D_{p} H(x, p)$ and define

$$
F(x)=\operatorname{co}\left\{f_{p}(x): p \in \mathbb{R}^{N} \backslash\{0\}\right\} \quad \forall x \in \mathbb{R}^{N},
$$

where 'co' stands for convex hull. Note that, for all $(x, p) \in \mathbb{R}^{N} \times\left(\mathbb{R}^{N} \backslash\{0\}\right)$,

$$
H(x, p)=\max _{v \in F(x)}\langle v, p\rangle \quad \text { and } \quad f_{p}(x)=\operatorname{argmax}_{v \in F(x)}\langle v, p\rangle .
$$

We begin by recovering properties of $F(\cdot)$ that follow directly from $(\mathrm{SA})$.

Lemma 3.1. The set-valued map $F$ is $2 \alpha$-Hölder continuous, i.e.,

$$
F(x) \subset F(y)+C_{0}|x-y|^{2 \alpha} B \quad \forall x, y \in \mathbb{R}^{N},
$$

and satisfies, for all $x \in \mathbb{R}^{N}$, the controllability condition

$$
B(0, r) \subset F(x) \subset B(0, R)
$$


as well as the curvature estimates

$$
B\left(f_{p}(x)-r \frac{p}{|p|}, r\right) \subset F(x) \quad \text { and } \quad F(x) \subset B\left(f_{p}(x)-R \frac{p}{|p|}, R\right) \quad \forall p \neq 0 .
$$

Remark 3.2. The first inclusion in (11) — which can be interpreted as an upper bound for the curvature of $\partial F(x)$ - is equivalent to the inequality

$$
\left|v-f_{p}(x)+r \frac{p}{|p|}\right| \geq r \quad \forall v \in \partial F(x),
$$

which in turn can be recast as follows

$$
-\frac{1}{2 r}\left|v-f_{p}(x)\right|^{2} \leq\left\langle v-f_{p}(x), \frac{p}{|p|}\right\rangle \quad \forall v \in \partial F(x) .
$$

The second inclusion in (11) - a lower bound for the curvature - can be rephrased as

$$
\left|v-f_{p}(x)+R \frac{p}{|p|}\right| \leq R \quad \forall v \in F(x),
$$

which is equivalent to

$$
\left\langle v-f_{p}(x), \frac{p}{|p|}\right\rangle \leq-\frac{1}{2 R}\left|v-f_{p}(x)\right|^{2} \quad \forall v \in F(x),
$$

or, since $F(x)$ is convex,

$$
\left\langle v-f_{p}(x), \frac{p}{|p|}\right\rangle \leq-\frac{1}{2 R}\left|v-f_{p}(x)\right|^{2} \quad \forall v \in \partial F(x) .
$$

Proof of Lemma 3.1. Note that, since $H(x, \cdot)$ is the support function of $F(x)$, inequality (3) directly implies (9) while (4) entails (10). Let us now check that the regularity condition (5) implies (11). For this we just have to note that, for any $v \in \partial F(x)$, there is some $q \neq 0$ such that $v=f_{q}(x)$, so that (5) becomes

$$
-\frac{1}{r}\left|v-f_{p}(x)\right|^{2} \leq\left\langle v-f_{p}(x), \frac{p}{|p|}\right\rangle \leq-\frac{1}{R}\left|v-f_{p}(x)\right|^{2} \quad \forall v \in \partial F(x) .
$$

These two equalities are equivalent to (12) and (13).

Next, we derive a regularity result for $f_{p}(\cdot)$, which is actually a consequence of the Hölder continuity of $F$ in (9) combined with the lower curvature bound in (5).

Lemma 3.3. For all $p \in \mathbb{R}^{N} \backslash\{0\}$ we have

$$
\left|f_{p}(x)-f_{p}(y)\right| \leq\left(C_{0}+\sqrt{2 C_{0} R}\right)|x-y|^{\alpha} \quad \forall x, y \in \mathbb{R}^{N} .
$$

Proof. Let $x, y \in \mathbb{R}^{N}$. In view of (9) there are $v_{y} \in F(x)$ and $v_{x} \in F(y)$ such that

$$
\left|f_{p}(y)-v_{y}\right| \leq C_{0}|x-y|^{2 \alpha} \quad \text { and } \quad\left|f_{p}(x)-v_{x}\right| \leq C_{0}|x-y|^{2 \alpha}
$$


Then, by (13),

$$
\begin{aligned}
& \left\langle v_{y}-f_{p}(x), \frac{p}{|p|}\right\rangle \leq-\frac{1}{2 R}\left|v_{y}-f_{p}(x)\right|^{2} \text { and }\left\langle v_{x}-f_{p}(y), \frac{p}{|p|}\right\rangle \\
& \quad \leq-\frac{1}{2 R}\left|v_{x}-f_{p}(y)\right|^{2} .
\end{aligned}
$$

Adding up the above two inequalities yields

$$
\begin{aligned}
& \left|v_{y}-f_{p}(x)\right|^{2}+\left|v_{x}-f_{p}(y)\right|^{2} \leq-2 R\left\langle v_{y}-f_{p}(x)+v_{x}-f_{p}(y), \frac{p}{|p|}\right\rangle \\
& \quad \leq 2 C_{0} R|x-y|^{2 \alpha} .
\end{aligned}
$$

So,

$$
\left|f_{p}(y)-f_{p}(x)\right| \leq\left|f_{p}(y)-v_{y}\right|+\left|v_{y}-f_{p}(x)\right| \leq\left(C_{0}+\sqrt{2 C_{0} R}\right)|x-y|^{\alpha},
$$

and the proof is complete.

The dependence of $f_{p}(x)$ with respect to $p$ is the object of our next result.

Lemma 3.4. For every $x \in \mathbb{R}^{N}$ we have

$$
\frac{1}{R}\left|f_{p}(x)-f_{q}(x)\right| \leq\left|\frac{p}{|p|}-\frac{q}{|q|}\right| \leq \frac{1}{r}\left|f_{p}(x)-f_{q}(x)\right|, \quad \forall p, q \in \mathbb{R}^{N} \backslash\{0\} .
$$

Proof. Let $x \in \mathbb{R}^{N}$ and let $p, q \in \mathbb{R}^{N} \backslash\{0\}$. Let us start with the first inequality. Recalling the second condition in (11) in its equivalent form (13), we have, since $f_{q}(x) \in \partial F(x)$,

$$
\left\langle f_{q}(x)-f_{p}(x), \frac{p}{|p|}\right\rangle \leq-\frac{1}{2 R}\left|f_{q}(x)-f_{p}(x)\right|^{2} .
$$

In a symmetric way we also have

$$
\left\langle f_{p}(x)-f_{q}(x), \frac{q}{|q|}\right\rangle \leq-\frac{1}{2 R}\left|f_{p}(x)-f_{q}(x)\right|^{2} .
$$

Adding the two estimates above easily gives the first inequality of (14) by Cauchy-Schwarz.

We now prove the second inequality, which is slightly more subtle. Recalling the first inclusion in (11) and the definition of $f_{p}(x)$, we conclude that

$$
\left\langle q, f_{p}(x)-r \frac{p}{|p|}+r b\right\rangle \leq\left\langle q, f_{q}(x)\right\rangle, \quad \forall b \in B .
$$

Hence,

$$
-r\left\langle\frac{q}{|q|}, \frac{p}{|p|}\right\rangle+r \leq\left\langle f_{q}(x)-f_{p}(x), \frac{q}{|q|}\right\rangle .
$$

Thus, exchanging $p$ and $q$,

$$
-r\left\langle\frac{p}{|p|}, \frac{q}{|q|}\right\rangle+r \leq\left\langle f_{p}(x)-f_{q}(x), \frac{p}{|p|}\right\rangle .
$$


Adding the above inequalities together leads to

$$
r\left|\frac{p}{|p|}-\frac{q}{|q|}\right|^{2}=2 r\left(1-\left\langle\frac{p}{|p|}, \frac{q}{|q|}\right\rangle\right) \leq\left\langle f_{p}(x)-f_{q}(x), \frac{p}{|p|}-\frac{q}{|q|}\right\rangle .
$$

Since $f_{p}(x)$ and $f_{q}(x)$ are boundary points, (12) yields

$$
\left\langle f_{p}(x)-f_{q}(x), \frac{p}{|p|}\right\rangle \leq \frac{1}{2 r}\left|f_{q}(x)-f_{p}(x)\right|^{2} .
$$

and

$$
\left\langle f_{q}(x)-f_{p}(x), \frac{q}{|q|}\right\rangle \leq \frac{1}{2 r}\left|f_{q}(x)-f_{p}(x)\right|^{2} .
$$

Therefore,

$$
\left\langle f_{p}(x)-f_{q}(x), \frac{p}{|p|}-\frac{q}{|q|}\right\rangle \leq \frac{1}{r}\left|f_{p}(x)-f_{q}(x)\right|^{2} .
$$

The conclusion follows from (15) and (16).

Let us now consider the polar of $H$, namely the function $H^{0}$ defined by

$$
H^{0}(x, q):=\max \{\langle p, q\rangle: H(x, p) \leq 1\} \quad \forall(x, q) \in \mathbb{R}^{N} \times \mathbb{R}^{N} .
$$

It is well-known that, for all $(x, q) \in \mathbb{R}^{N} \times \mathbb{R}^{N}$,

$$
H^{0}(x, q) \leq 1 \quad \Longleftrightarrow \quad q \in F(x),
$$

and

$$
H^{0}\left(x, D_{p} H(x, p)\right)=H^{0}\left(x, f_{p}(x)\right)=1 \quad \forall(x, p) \in \mathbb{R}^{N} \times\left(\mathbb{R}^{N} \backslash\{0\}\right) .
$$

The duality between $H$ and $H^{0}$ brings similar qualitative properties for these two functions. For instance, on account of (10), we have

$$
\frac{|q|}{R} \leq H^{0}(x, q) \leq \frac{|q|}{r} \quad \forall(x, q) \in \mathbb{R}^{N} \times \mathbb{R}^{N} .
$$

Moreover, $H^{0}$ is also Hölder continuous with respect to $x$, with the same exponent as $H$.

Lemma 3.5. For all $q \in \mathbb{R}^{N}$,

$$
\left|H^{0}(x, q)-H^{0}(y, q)\right| \leq \frac{C_{0}}{r^{2}}|q||x-y|^{2 \alpha} \quad \forall x, y \in \mathbb{R}^{N} .
$$

Proof. Let $x, y, q \in \mathbb{R}^{N}$. Take $p \in \mathbb{R}^{N}$, with $H(x, p) \leq 1$, such that $H^{0}(x, q)=$ $\langle p, q\rangle$. Then, by (4), $|p| \leq 1 / r$. Also, by (3),

$$
H(y, p) \leq 1+\frac{C_{0}}{r}|x-y|^{2 \alpha} .
$$

So,

$$
H^{0}(y, q) \geq\left\langle\frac{p}{1+\frac{C_{0}}{r}|x-y|^{2 \alpha}}, q\right\rangle=\frac{H^{0}(x, q)}{1+\frac{C_{0}}{r}|x-y|^{2 \alpha}} .
$$


On the other hand, in view of (19),

$$
\begin{aligned}
H^{0}(x, q) & =\frac{H^{0}(x, q)}{1+\frac{C_{0}}{r}|x-y|^{2 \alpha}}+\frac{\frac{C_{0}}{r}|x-y|^{2 \alpha}}{1+\frac{C_{0}}{r}|x-y|^{2 \alpha}} H^{0}(x, q) \\
& \leq \frac{H^{0}(x, q)}{1+\frac{C_{0}}{r}|x-y|^{2 \alpha}}+\frac{C_{0}}{r^{2}}|q||x-y|^{2 \alpha} .
\end{aligned}
$$

Thus,

$$
H^{0}(y, q) \geq H^{0}(x, q)-\frac{C_{0}}{r^{2}}|q \| x-y|^{2 \alpha} .
$$

Hence, we obtain the conclusion exchanging the roles of $x$ and $y$.

We now turn to the analysis of the level set

$$
F^{0}(x)=\left\{p \in \mathbb{R}^{N}: H(x, p) \leq 1\right\} \quad x \in \mathbb{R}^{N} .
$$

Lemma 3.6. Let $x \in \mathbb{R}^{N}$. Then, for every $p, p^{\prime} \in \mathbb{R}^{N}$ with $H(x, p)=H\left(x, p^{\prime}\right)=1$,

$$
\left|p-p^{\prime}\right| \leq C\left|\frac{p^{\prime}}{\left|p^{\prime}\right|}-\frac{p}{|p|}\right|
$$

for some constant $C$.

Proof. First of all, the reader be warned that, as $x$ plays no role in this proof, the $x$-dependence in $H$ will be omitted. For all $\theta, \theta^{\prime} \in S^{N-1}$, we have

$$
\left|\frac{\theta}{H(\theta)}-\frac{\theta^{\prime}}{H\left(\theta^{\prime}\right)}\right| \leq \frac{\left|\theta-\theta^{\prime}\right|}{H(\theta)}+\frac{\left|H(\theta)-H\left(\theta^{\prime}\right)\right|}{H(\theta) H\left(\theta^{\prime}\right)} .
$$

Since $H$ is Lipschitz continuous by (10), recalling $r \leq H(\theta), H\left(\theta^{\prime}\right) \leq R$ we conclude that

$$
\frac{\left|\theta-\theta^{\prime}\right|}{H(\theta)}+\frac{\left|H(\theta)-H\left(\theta^{\prime}\right)\right|}{H(\theta) H\left(\theta^{\prime}\right)} \leq C\left|\theta-\theta^{\prime}\right|
$$

for some constant $C$. Therefore,

$$
\left|\frac{\theta}{H(\theta)}-\frac{\theta^{\prime}}{H\left(\theta^{\prime}\right)}\right| \leq C\left|\theta-\theta^{\prime}\right|
$$

Now, observe that the map $\theta \mapsto \theta / H(\theta)$ is a bijection between the unit sphere $S^{N-1}$ and $\partial F^{0}(x)$. So, applying the above inequality to $\theta, \theta^{\prime} \in S^{N-1}$, which we now choose such that $p=\theta / H(\theta)$ and $p^{\prime}=\theta^{\prime} / H\left(\theta^{\prime}\right)$, the conclusion follows.

Lemma 3.7. (Lower curvature estimate for $F^{0}$ ) There is a constant $R^{\prime}$ such that $F^{0}(x)$ satisfies the lower curvature estimate of radius $R^{\prime}$ for all $x \in \mathbb{R}^{N}$, i.e.,

$$
F^{0}(x) \subset B\left(\frac{p}{H(x, p)}-R^{\prime} \frac{f_{p}(x)}{\left|f_{p}(x)\right|}, R^{\prime}\right) \quad \forall x, p \in \mathbb{R}^{N}, p \neq 0
$$

or, equivalently,

$$
\left\langle p^{\prime}-p, \frac{f_{p}}{\left|f_{p}\right|}\right\rangle \leq-\frac{1}{2 R^{\prime}}\left|p^{\prime}-p\right|^{2} \quad \forall p, p^{\prime} \in \partial F^{0}
$$


Proof. Again, we shall drop $x$-dependence in all the formulas below since it is of no interest for this proof. Recalling Remark 3.2 we conclude that it suffices to prove inequality (22) for some constant $R^{\prime}$. Let then $p, p^{\prime} \in \partial F^{0}$. Since $H$ is positively homogeneous of degree 1 , we have

$$
\begin{aligned}
& H\left(p^{\prime}\right)-H(p)-\left\langle D_{p} H(p), p^{\prime}-p\right\rangle=\left\langle D_{p} H\left(p^{\prime}\right), p^{\prime}\right\rangle-\left\langle D_{p} H(p), p\right\rangle \\
& \quad-\left\langle D_{p} H(p), p^{\prime}-p\right\rangle=\left\langle D_{p} H\left(p^{\prime}\right)-D_{p} H(p), p^{\prime}\right\rangle
\end{aligned}
$$

where $D_{p} H(p)=f_{p}$ and $D_{p} H\left(p^{\prime}\right)=f_{p^{\prime}}$. From the lower curvature estimate on $F$ given in (13) it follows that

$$
\left\langle f_{p}-f_{p^{\prime}}, \frac{p^{\prime}}{\left|p^{\prime}\right|}\right\rangle \leq-\frac{1}{2 R}\left|f_{p^{\prime}}-f_{p}\right|^{2} .
$$

Thus, combining the above inequality with the previous identity, and using the fact that $H(p)=H\left(p^{\prime}\right)=1$,

$$
\left\langle f_{p}, p^{\prime}-p\right\rangle \leq-\frac{\left|p^{\prime}\right|}{2 R}\left|f_{p^{\prime}}-f_{p}\right|^{2} .
$$

Now, apply Lemma 3.4 to obtain

$$
\left\langle f_{p}, p^{\prime}-p\right\rangle \leq-\frac{r^{2}\left|p^{\prime}\right|}{2 R}\left|\frac{p^{\prime}}{\left|p^{\prime}\right|}-\frac{p}{|p|}\right|^{2}
$$

Finally, let $C$ be the constant given by Lemma 3.6. Then, (21) and (23) yield

$$
\left\langle\frac{f_{p}}{\left|f_{p}\right|}, p^{\prime}-p\right\rangle \leq-\frac{r^{2}\left|p^{\prime}\right|}{2 R^{2}}\left|\frac{p^{\prime}}{\left|p^{\prime}\right|}-\frac{p}{|p|}\right|^{2} \leq-\frac{r^{2}}{2 C^{2} R^{3}}\left|p^{\prime}-p\right|^{2} .
$$

whence the conclusion follows with $R^{\prime}=C^{2} R^{3} / r^{2}$.

In particular, Lemma 3.7 ensures $F^{0}(x)$ is a strictly convex set for any $x \in \mathbb{R}^{N}$. Thus, since $H^{0}(x, \cdot)$ is the support function of $F^{0}(x), D_{q} H^{0}(x, q)$ exists for any $x, q \in \mathbb{R}^{N}$ with $q \neq 0$ (see, for instance, [3, Theorem A.1.20]). In fact, we shall soon prove a stronger property: the map $q \rightarrow D_{q} H^{0}(x, q)$ is locally Lipschitz continuous in $\mathbb{R}^{N} \backslash\{0\}$. Before doing this, let us collect some technical remarks on the link between $H^{0}$ and $H$ and their derivatives.

Lemma 3.8. We have, for any $p, q \in \mathbb{R}^{N} \backslash\{0\}$,

$$
\left[q \in \partial F(x) \text { and } p=D_{q} H^{0}(x, q)\right] \quad \Longleftrightarrow \quad\left[p \in \partial F^{0}(x) \text { and } q=D_{p} H(x, p)\right]
$$

In particular,

$$
D_{q} H^{0}\left(x, \frac{f_{p}(x)}{\left|f_{p}(x)\right|}\right)=p \quad \forall p \in \partial F^{0}(x) .
$$

Proof. We just need to show the implication

$$
\left[q \in \partial F(x) \text { and } p=D_{q} H^{0}(x, q)\right] \Longrightarrow\left[p \in \partial F^{0}(x) \text { and } q=D_{p} H(x, p)\right]
$$

because $H^{00}=H$. Let $q \in \partial F(x)$ and $p=D_{q} H^{0}(x, q)$. Note that $H(x, p)=$ $H^{0}(x, q)=1$ and, in particular, $p \in \partial F^{0}(x)$. By definition, we have

$$
H^{0}\left(x, q^{\prime}\right) H\left(x, p^{\prime}\right) \geq\left\langle p^{\prime}, q^{\prime}\right\rangle \quad \forall p^{\prime}, q^{\prime} \in \mathbb{R}^{N} .
$$


This inequality becomes an equality for $\left(p^{\prime}, q^{\prime}\right)=(p, q)$ because

$$
\langle p, q\rangle=\left\langle D_{q} H^{0}(x, q), q\right\rangle=H^{0}(x, q)=1=H^{0}(x, q) H(x, p) .
$$

Taking the derivative in (26) with respect to $p$ then gives

$$
H^{0}(x, q) D_{p} H(x, p)=D_{p} H(x, p)=q .
$$

Next, we turn to the proof of (25). Recall first that $f_{p}(x)=D_{p} H(x, p)$ for any $p \neq 0$. So, if $p \in \partial F^{0}(x)$, then (24) implies that

$$
D_{q} H^{0}\left(x, \frac{f_{p}(x)}{\left|f_{p}(x)\right|}\right)=D_{q} H^{0}\left(x, f_{p}(x)\right)=p,
$$

since $D_{q} H^{0}(x, \cdot)$ is 0 -homogeneous.

Lemma 3.9. There is a constant $C$ such that, for every $x \in \mathbb{R}^{N}$,

$$
\left|D_{q} H^{0}(x, q)-D_{q} H^{0}\left(x, q^{\prime}\right)\right| \leq \frac{C}{|q| \vee\left|q^{\prime}\right|}\left|q-q^{\prime}\right| \quad \forall q, q^{\prime} \in \mathbb{R}^{N} \backslash\{0\} .
$$

Proof. Let us fix $p, p^{\prime} \in \partial F^{0}(x)$. Owing to Lemma 3.7 in its equivalent form (22), we deduce that

$$
\left\langle p^{\prime}-p, \frac{f_{p}(x)}{\left|f_{p}(x)\right|}\right\rangle \leq-\frac{1}{2 R^{\prime}}\left|p^{\prime}-p\right|^{2}
$$

and

$$
\left\langle p-p^{\prime}, \frac{f_{p^{\prime}}(x)}{\left|f_{p^{\prime}}(x)\right|}\right\rangle \leq-\frac{1}{2 R^{\prime}}\left|p^{\prime}-p\right|^{2} .
$$

Adding up the last two inequalities, we obtain

$$
\left|p^{\prime}-p\right|^{2} \leq R^{\prime}\left\langle p^{\prime}-p, \frac{f_{p^{\prime}}(x)}{\left|f_{p^{\prime}}(x)\right|}-\frac{f_{p}(x)}{\left|f_{p}(x)\right|}\right\rangle \leq R^{\prime}\left|p^{\prime}-p\right|\left|\frac{f_{p^{\prime}}(x)}{\left|f_{p^{\prime}}(x)\right|}-\frac{f_{p}(x)}{\left|f_{p}(x)\right|}\right| .
$$

Now, recall that the map $p \mapsto f_{p}(x) /\left|f_{p}(x)\right|$ is a bijection from $\partial F^{0}(x)$ to $S^{N-1}$ to deduce that for all $q, q^{\prime} \in S^{N-1}$ there are $p, p^{\prime} \in \partial F^{0}(x)$ such that $q=f_{p}(x) /\left|f_{p}(x)\right|$ and $q^{\prime}=f_{p^{\prime}}(x) /\left|f_{p^{\prime}}(x)\right|$. Then, combining (25) and (27),

$$
\begin{aligned}
\left|D_{q} H^{0}\left(x, q^{\prime}\right)-D_{q} H^{0}(x, q)\right| & =\left|D_{q} H^{0}\left(x, \frac{f_{p^{\prime}}(x)}{\left|f_{p^{\prime}}(x)\right|}\right)-D_{q} H^{0}\left(x, \frac{f_{p}(x)}{\left|f_{p}(x)\right|}\right)\right| \\
& =\left|p^{\prime}-p\right| \\
& \leq R^{\prime}\left|\frac{f_{p^{\prime}}(x)}{\left|f_{p^{\prime}}(x)\right|}-\frac{f_{p}(x)}{\left|f_{p}(x)\right|}\right|=R^{\prime}\left|q^{\prime}-q\right| .
\end{aligned}
$$

This is the desired estimate for $q, q^{\prime} \in S^{N-1}$. Next, let $q, q^{\prime} \in \mathbb{R}^{N} \backslash\{0\}$. Then, since $D_{q} H^{0}(x, \cdot)$ is homogeneous of degree 0 ,

$$
\left|D_{q} H^{0}\left(x, q^{\prime}\right)-D_{q} H^{0}(x, q)\right| \leq R^{\prime}\left|\frac{q^{\prime}}{\left|q^{\prime}\right|}-\frac{q}{|q|}\right| .
$$


Finally, observe that

$$
\begin{aligned}
\left|\frac{q^{\prime}}{\left|q^{\prime}\right|}-\frac{q}{|q|}\right| & \leq\left|\frac{q^{\prime}}{\left|q^{\prime}\right|}-\frac{q}{\left|q^{\prime}\right|}\right|+\left|\frac{q}{\left|q^{\prime}\right|}-\frac{q}{|q|}\right|=\frac{\left|q^{\prime}-q\right|}{\left|q^{\prime}\right|}+\frac{|q|}{|q|\left|q^{\prime}\right|}|| q^{\prime}|-| q|| \\
& \leq 2 \frac{\left|q^{\prime}-q\right|}{\left|q^{\prime}\right|}
\end{aligned}
$$

to complete the proof.

\section{Regularity of extremal trajectories}

In this section, we shall prove a regularity result for the extremal trajectories of the differential inclusion

$$
x^{\prime}(t) \in F(x(t)) \quad t \geq 0,
$$

where $F$ is the multifunction introduced in (7), and $H$ is a given function satisfying (SA). Alternatively, this analysis could be addressed to differential inclusions associated with a multifunction $F: \mathbb{R}^{N} \rightrightarrows \mathbb{R}^{N}$ that satisfies (9), (11), and (10) as standing assumptions, in which case the Hamiltonian $H$ should be defined as in (8).

A trajectory of the above differential inclusion is a locally absolutely continuous arc $x(\cdot):[0, \infty) \rightarrow \mathbb{R}^{N}$ that satisfies (28) for a.e. $t \geq 0$. Given a closed subset $K$ of $\mathbb{R}^{N}$, we denote by $\mathcal{R}(t), t \geq 0$, the reachable set (from $K$ ) in time $t$, that is,

$$
\mathcal{R}(t)=\{x(t): x(\cdot) \text { is a trajectory of }(28) \text { with } x(0) \in K\} .
$$

A trajectory $\bar{x}(\cdot)$ of $(28)$ is called extremal on the time interval $[0, t]$ if $\bar{x}(t) \in$ $\partial \mathcal{R}(t)$. In this case, one can show that in fact $\bar{x}(s) \in \mathcal{R}(s)$ for every $s \in[0, t]$.

Due to the special structure of $F$, described by the properties (9), (11), and (10), we will be able to show that all extremal trajectories are $\mathcal{C}^{1, \alpha / 2}$ smooth. More precisely, we have the following result.

Theorem 4.1. Assume (SA) and let $\bar{x}$ be an extremal trajectory of (28) on some time interval $[0, T]$. Then

$$
\left|\bar{x}^{\prime}\left(t_{2}\right)-\bar{x}^{\prime}\left(t_{1}\right)\right| \leq C\left(t_{2}-t_{1}\right)^{\alpha / 2} \quad \forall t_{1}, t_{2} \in[0, T]
$$

for some constant $C$.

Proof. Let $\bar{x}$ be an extremal trajectory on $[0, T]$. Then, by extremality, $x^{\prime}(t) \in$ $\partial F(\bar{x}(t))$ for almost all $t \in[0, T]$, so that we can set

$$
\bar{p}(t)=D_{q} H^{0}\left(\bar{x}(t), \bar{x}^{\prime}(t)\right) \quad \text { a.e. in }[0, T] .
$$

Using Lemma 3.8, we obtain the following relation between $\bar{x}$ and $\bar{p}$ :

$$
\bar{x}^{\prime}(t)=D_{p} H(\bar{x}(t), \bar{p}(t)) \quad \text { for a.e. } \quad t \in[0, T] .
$$

Step 1. We first claim that, for any $0 \leq t_{1}<t_{2} \leq T$ we have

$$
t_{2}-t_{1} \leq H^{0}\left(\bar{x}\left(t_{2}\right), \int_{t_{1}}^{t_{2}} D_{p} H\left(\bar{x}\left(t_{2}\right), \bar{p}(t)\right) d t\right)+C\left(t_{2}-t_{1}\right)^{1+\alpha} .
$$


Proof of (30) Let us set

$$
q=\frac{\bar{x}\left(t_{2}\right)-\bar{x}\left(t_{1}\right)}{\left|\bar{x}\left(t_{2}\right)-\bar{x}\left(t_{1}\right)\right|}
$$

Let $\lambda:\left[t_{1}, t_{2}\right] \rightarrow \mathbb{R}$ be a solution of the Cauchy problem

$$
\left\{\begin{array}{l}
\lambda^{\prime}(t)=\frac{1}{H^{0}\left(\bar{x}\left(t_{1}\right)+\lambda(t) q, q\right)}, t \in\left[t_{1}, t_{2}\right] \\
\lambda\left(t_{1}\right)=0
\end{array}\right.
$$

Then $x(t):=\bar{x}\left(t_{1}\right)+\lambda(t) q$ is a trajectory of (28) since, owing to (17),

$$
H^{0}\left(x(t), x^{\prime}(t)\right)=H^{0}\left(x(t), \lambda^{\prime}(t) q\right)=\frac{H^{0}\left(\bar{x}\left(t_{1}\right)+\lambda(t) q, q\right)}{H^{0}\left(\bar{x}\left(t_{1}\right)+\lambda(t) q, q\right)}=1
$$

for all $t \in\left[t_{1}, t_{2}\right]$. Therefore, since $\bar{x}$ is an extremal trajectory, the point $\bar{x}\left(t_{1}\right)+$ $\lambda\left(t_{2}\right) q$ belongs to the segment $\left[\bar{x}\left(t_{1}\right), \bar{x}\left(t_{2}\right)\right]$. So,

$$
\lambda\left(t_{2}\right)-\lambda\left(t_{1}\right) \leq\left|\bar{x}\left(t_{2}\right)-\bar{x}\left(t_{1}\right)\right| .
$$

Note that, owing to (20), the above inequality, and the boundedness of $F$,

$$
\left|\frac{1}{H^{0}\left(\bar{x}\left(t_{1}\right)+\lambda(t) q, q\right)}-\frac{1}{H^{0}\left(\bar{x}\left(t_{2}\right), q\right)}\right| \leq C\left|\bar{x}\left(t_{2}\right)-\bar{x}\left(t_{1}\right)\right|^{2 \alpha} \leq C\left(t_{2}-t_{1}\right)^{2 \alpha}
$$

for all $t \in\left[t_{1}, t_{2}\right]$ and some constant $C$. Hence,

$$
\lambda\left(t_{2}\right)-\lambda\left(t_{1}\right)=\int_{t_{1}}^{t_{2}} \frac{d t}{H^{0}\left(\bar{x}\left(t_{1}\right)+\lambda(t) q, q\right)} \geq \frac{t_{2}-t_{1}}{H^{0}\left(\bar{x}\left(t_{2}\right), q\right)}-C\left(t_{2}-t_{1}\right)^{1+2 \alpha} .
$$

So, appealing to Lemma 3.3,

$$
\begin{aligned}
t_{2}-t_{1} & \leq H^{0}\left(\bar{x}\left(t_{2}\right), q\right)\left|\bar{x}\left(t_{2}\right)-\bar{x}\left(t_{1}\right)\right|+C\left(t_{2}-t_{1}\right)^{1+2 \alpha} \\
& =H^{0}\left(\bar{x}\left(t_{2}\right), \bar{x}\left(t_{2}\right)-\bar{x}\left(t_{1}\right)\right)+C\left(t_{2}-t_{1}\right)^{1+2 \alpha} \\
& =H^{0}\left(\bar{x}\left(t_{2}\right), \int_{t_{1}}^{t_{2}} D_{p} H(\bar{x}(t), \bar{p}(t)) d t\right)+C\left(t_{2}-t_{1}\right)^{1+2 \alpha} \\
& \leq H^{0}\left(\bar{x}\left(t_{2}\right), \int_{t_{1}}^{t_{2}} D_{p} H\left(\bar{x}\left(t_{2}\right), \bar{p}(t)\right) d t\right)+C\left(t_{2}-t_{1}\right)^{1+\alpha}
\end{aligned}
$$

where constants may change from line to line. We have thus proved (30).

Step 2. Let us fix $0 \leq t_{1}<t_{2} \leq T$ and let $\bar{t}$ be such that

$$
H^{0}\left(\bar{x}\left(t_{2}\right), \bar{x}(\bar{t})-\bar{x}\left(t_{1}\right)\right)=H^{0}\left(\bar{x}\left(t_{2}\right), \bar{x}\left(t_{2}\right)-\bar{x}(\bar{t})\right) .
$$

Define

$$
a=\bar{x}(\bar{t})-\bar{x}\left(t_{1}\right) \quad \text { and } \quad b=\bar{x}\left(t_{2}\right)-\bar{x}(\bar{t})
$$

We claim that

$$
H^{0}\left(\bar{x}\left(t_{2}\right), a\right)+H^{0}\left(\bar{x}\left(t_{2}\right), b\right) \leq H^{0}\left(\bar{x}\left(t_{2}\right), a+b\right)+C\left(t_{2}-t_{1}\right)^{1+\alpha}
$$

and

$$
\left|2 \bar{t}-t_{1}-t_{2}\right| \leq C\left(t_{2}-t_{1}\right)^{1+\alpha}
$$


Proof of (33) and (34) Again by Lemma 3.3, and then using Jensen's inequality, we obtain

$$
\begin{aligned}
H^{0}\left(\bar{x}\left(t_{2}\right), a\right) & =H^{0}\left(\bar{x}\left(t_{2}\right), \int_{t_{1}}^{\bar{t}} D_{p} H(\bar{x}(s), \bar{p}(s)) d s\right) \\
& \leq H^{0}\left(\bar{x}\left(t_{2}\right), \int_{t_{1}}^{\bar{t}} D_{p} H\left(\bar{x}\left(t_{2}\right), \bar{p}(s)\right) d s\right)+C\left(t_{2}-t_{1}\right)^{1+\alpha} \\
& \leq \int_{t_{1}}^{\bar{t}} H^{0}\left(\bar{x}\left(t_{2}\right), D_{p} H\left(\bar{x}\left(t_{2}\right), \bar{p}(s)\right)\right) d s+C\left(t_{2}-t_{1}\right)^{1+\alpha} \\
& \leq \bar{t}-t_{1}+C\left(t_{2}-t_{1}\right)^{1+\alpha} .
\end{aligned}
$$

Applying (30) between $t_{1}$ and $\bar{t}$ gives

$$
\bar{t}-t_{1} \leq H^{0}\left(\bar{x}(\bar{t}), \int_{t_{1}}^{\bar{t}} D_{p} H(\bar{x}(\bar{t}), \bar{p}(t)) d t\right)+C\left(\bar{t}-t_{1}\right)^{1+\alpha} .
$$

Now, in order to bound the above right-hand side observe that

$$
\left|D_{p} H(\bar{x}(\bar{t}), \bar{p}(t))-D_{p} H(\bar{x}(t), \bar{p}(t))\right| \leq C|\bar{x}(\bar{t})-\bar{x}(t)|^{\alpha} \leq C\left(\bar{t}-t_{1}\right)^{\alpha}
$$

in view of Lemma 3.3, and

$$
\left|H^{0}(\bar{x}(\bar{t}), a)-H^{0}\left(\bar{x}\left(t_{2}\right), a\right)\right| \leq C|a|\left|\bar{x}(\bar{t})-\bar{x}\left(t_{2}\right)\right|^{2 \alpha} \leq C\left(\bar{t}_{2}-t_{1}\right)^{1+2 \alpha}
$$

owing to Lemma 3.5. Therefore, (36) leads to

$$
\begin{aligned}
\bar{t}-t_{1} & \leq H^{0}(\bar{x}(\bar{t}), \underbrace{\int_{t_{1}}^{\bar{t}} D_{p} H(\bar{x}(t), \bar{p}(t)) d t}_{a})+C\left(\bar{t}-t_{1}\right)^{1+\alpha} \\
& \leq H^{0}\left(\bar{x}\left(t_{2}\right), a\right)+C\left(t_{2}-t_{1}\right)^{1+\alpha} .
\end{aligned}
$$

On account of (35) and (37), we have

$$
H^{0}\left(\bar{x}\left(t_{2}\right), a\right)-C\left(t_{2}-t_{1}\right)^{1+\alpha} \leq \bar{t}-t_{1} \leq H^{0}\left(\bar{x}\left(t_{2}\right), a\right)+C\left(t_{2}-t_{1}\right)^{1+\alpha} .
$$

In the same way,

$$
H^{0}\left(\bar{x}\left(t_{2}\right), b\right)-C\left(t_{2}-t_{1}\right)^{1+\alpha} \leq t_{2}-\bar{t} \leq H^{0}\left(\bar{x}\left(t_{2}\right), b\right)+C\left(t_{2}-t_{1}\right)^{1+\alpha}
$$

Combining the above two inequalities with the choice of $\bar{t}$ made in (31) gives (34). Moreover, adding up the above inequalities and recalling (30), we get 


$$
\begin{aligned}
H^{0}\left(\bar{x}\left(t_{2}\right), a\right)+H^{0}\left(\bar{x}\left(t_{2}\right), b\right) & \leq\left(t_{2}-t_{1}\right)+C\left(t_{2}-t_{1}\right)^{1+\alpha} \\
& \leq H^{0}\left(\bar{x}\left(t_{2}\right), a+b\right)+C\left(t_{2}-t_{1}\right)^{1+\alpha},
\end{aligned}
$$

which yields (33).

Step 3. We now claim that, for any $0 \leq t_{1}<t_{2} \leq T$, we have

$$
\left|\bar{x}\left(\frac{t_{1}+t_{2}}{2}\right)-\frac{\bar{x}\left(t_{2}\right)+\bar{x}\left(t_{1}\right)}{2}\right| \leq C\left(t_{2}-t_{1}\right)^{1+\alpha} .
$$

Proof of (38) Having fixed $0 \leq t_{1}<t_{2} \leq T$, we will use the same notation for $\bar{t}, a$, and $b$ as in (31) and (32). Moreover, since $x\left(t_{2}\right)$ is fixed in the reasoning below, as we often did before we will omit the $x\left(t_{2}\right)$-dependance of $H^{0}$ and all other maps appearing in this proof.

Let us set, for any $q \in \mathbb{R}^{N} \backslash\{0\}, g_{q}=D_{q} H^{0}(q)$. We use below repetitively the following remark:

$$
\text { for any } q \neq 0 \text {, if } p=g_{q}, \quad \text { then } f_{p}=q / H^{0}(q) \text {. }
$$

Indeed, since $q / H^{0}(q) \in \partial F$ and $p=D_{q} H^{0}\left(q / H^{0}(q)\right)\left(D_{q} H^{0}\right.$ is 0-homogeneous), Lemma 3.8 implies that $q / H^{0}(q)=D_{p} H(p)=f_{p}$.

We first show that

$$
\frac{1}{2 R^{\prime}}\left|g_{q}-g_{q^{\prime}}\right|^{2} \leq\left\langle g_{q}-g_{q^{\prime}}, \frac{q}{|q|}\right\rangle \quad \forall q, q^{\prime} \in \mathbb{R}^{N} \backslash\{0\},
$$

where $R^{\prime}$ is the constant appearing in Lemma 3.7. For this, let us consider the lower curvature estimate (22) in Lemma 3.7 with $p=g_{q}$ and $p^{\prime}=g_{q^{\prime}}$ : because of the remark above and since $p, p^{\prime} \in \partial F^{0}$, we have

$$
\left\langle g_{q^{\prime}}-g_{q}, \frac{q}{|q|}\right\rangle \leq-\frac{1}{2 R^{\prime}}\left|g_{q^{\prime}}-g_{q}\right|^{2}
$$

which is exactly (39).

Next, we note that

$$
|a-b| \leq C H^{0}(a)\left|g_{a}-g_{b}\right| .
$$

Indeed, let us apply the first inequality in Lemma 3.4 to $p=g_{a}$ and $q=g_{b}$. Since $f_{p}=a / H^{0}(a), f_{q}=b / H^{0}(b)$, and-by $(31)-H^{0}(a)=H^{0}(b)$, we have

$$
|a-b| \leq R H^{0}(a)\left|\frac{g_{a}}{\left|g_{a}\right|}-\frac{g_{b}}{\left|g_{b}\right|}\right| \leq C H^{0}(a)\left|g_{a}-g_{b}\right| .
$$

In order to estimate the right-hand side of inequality (40), let us observe that, in view of $(33)$,

$$
\begin{aligned}
0 & \leq H^{0}(a+b)-H^{0}(a)-H^{0}(b)+C\left(t_{2}-t_{1}\right)^{1+\alpha} \\
& =\left\langle g_{a+b}, a+b\right\rangle-\left\langle g_{a}, a\right\rangle-\left\langle g_{b}, b\right\rangle+C\left(t_{2}-t_{1}\right)^{1+\alpha}
\end{aligned}
$$

so that

$$
0 \leq\left\langle g_{a+b}-g_{a}, a\right\rangle+\left\langle g_{a+b}-g_{b}, b\right\rangle+C\left(t_{2}-t_{1}\right)^{1+\alpha} .
$$

Plugging inequality (39) into this inequality leads to

$$
|a|\left|g_{a+b}-g_{a}\right|^{2}+|b|\left|g_{a+b}-g_{b}\right|^{2} \leq C\left(t_{2}-t_{1}\right)^{1+\alpha} .
$$


Now, since $|a| \geq\left(\bar{t}-t_{1}\right) / C$ and $|b| \geq\left(t_{2}-\bar{t}\right) / C$, in view of (34) we have

$$
\left|g_{a+b}-g_{a}\right| \leq C\left(t_{2}-t_{1}\right)^{\alpha / 2}, \quad\left|g_{a+b}-g_{b}\right| \leq C\left(t_{2}-t_{1}\right)^{\alpha / 2} .
$$

Also, since $H^{0}(a) \leq C\left(\bar{t}-t_{1}\right) \leq C\left(t_{2}-t_{1}\right)$, from (40) we get

$$
|a-b| \leq C H^{0}(a)\left(\left|g_{a+b}-g_{a}(x)\right|+\left|g_{a+b}-g_{b}(x)\right|\right) \leq C\left(t_{2}-t_{1}\right)^{1+\alpha / 2} .
$$

Recalling the definition of $a$ and $b$, this means that

$$
\left|2 \bar{x}(\bar{t})-\bar{x}\left(t_{1}\right)-\bar{x}\left(t_{2}\right)\right| \leq C\left(t_{2}-t_{1}\right)^{1+\alpha / 2} .
$$

Using again (34) and the Lipschitz continuity of $\bar{x}$, the above inequality yields (38).

Conclusion. In view of (38), Theorem 2.1.10 of [3] states that each component of $\bar{x}$ is semi-convex and semi-concave with a modulus $m$ of the form $m(\rho)=C \rho^{\alpha / 2}$. Then, from Theorem 3.3.7 of [3], $\bar{x}$ is of class $\mathcal{C}^{1, \alpha / 2}$ and (29) holds.

\section{The semiconcavity result}

Let $H: \mathbb{R}^{N} \times \mathbb{R}^{N} \rightarrow \mathbb{R}$ be a continuous function satisfying our Standing Assumptions with constants $\alpha, r, R$, and $C_{0}$, and let $\Omega \subset \mathbb{R}^{N}$ be an open set.

In this section, we will apply the previous analysis to study the regularity of the solution to the Dirichlet problem

$$
\begin{cases}H(x,-D u(x))=1 & \text { in } \Omega \\ u(x)=0 & \text { on } \partial \Omega\end{cases}
$$

The existence, uniqueness, and Lipschitz continuity of the viscosity solution $u$ of the above problem is well-known, as well as the representation formula

$$
u(x)=\inf \{t \geq 0: \exists x(\cdot) \text { trajectory of }(28) \text { with } x(0)=x, x(t) \in \partial \Omega\}
$$

(see, e.g., [1]).

We recall that a function $v: \Omega \rightarrow \mathbb{R}$ is locally $\theta$-semiconcave, with $\theta \in$ $(0,1]$, if for every compact convex set $\mathcal{O} \subset \Omega$ there is a constant $C_{\mathcal{O}}$ such that

$$
v(\lambda x+(1-\lambda) y) \geq \lambda v(x)+(1-\lambda) v(y)-C_{\mathcal{O}} \lambda(1-\lambda)|x-y|^{1+\theta}
$$

for all $x, y \in \mathcal{O}$ and $\lambda \in[0,1]$. We are now ready for our main result.

Theorem 5.1. Assume (SA). Then the solution $u$ of (41) is locally $\theta$-semiconcave in $\Omega$ for every $\theta \in\left(0, \frac{\alpha}{4+\alpha}\right)$.

Proof. The strategy of the proof is the following. Fix

$$
\beta \in\left(\frac{2}{2+\alpha}, \frac{4}{4+\alpha}\right),
$$

and observe that

$$
0<\theta:=\beta \frac{2+\alpha}{2}-1<\frac{\alpha}{4+\alpha} .
$$

Let $\mathcal{O} \subset \subset \Omega$ be an open convex set. We are going to show that

$$
u(x+h)+u(x-h)-2 u(x) \leq C|h|^{\beta(2+\alpha) / 2}
$$


for all $h \in \mathbb{R}^{N}$ sufficiently small (in this proof, $C$ denotes a generic constant depending only on $\alpha, r, R, C_{0}$, and $\mathcal{O}$ ). Since $u$ is continuous, owing to [3, Theorem 2.1.10] the above inequality implies that $u$ is locally $\theta$-semiconcave in $\Omega$.

Step 1. Let $\bar{x} \in \mathcal{O}$ and let $\bar{x}(\cdot)$ be a solution of the minimization problem in (42) - an optimal trajectory for short. Since $\bar{x}(\cdot)$ is extremal on $[0, u(\bar{x})]$, Theorem 4.1 implies that $\bar{x}(\cdot)$ is of class $\mathcal{C}^{1, \alpha / 2}$ and satisfies

$$
\left|\bar{x}^{\prime}\left(t_{2}\right)-\bar{x}^{\prime}\left(t_{1}\right)\right| \leq C\left|t_{2}-t_{1}\right|^{\alpha / 2} \quad \forall t_{1}, t_{2} \in[0, u(\bar{x})] .
$$

Setting $\bar{v}=\bar{x}^{\prime}(0)$, from the above inequality we obtain

$$
|\bar{x}(t)-\bar{x}-t \bar{v}| \leq C t^{1+\alpha / 2} \quad \forall t \in[0, u(\bar{x})] .
$$

Step 2. Let $h \in \mathbb{R}^{N}$ be small enough, and set $\bar{t}=|h|^{\beta}$. We will now build a trajectory $x_{+}(\cdot)$ and a time $\tau_{+}$such that

$$
\left\{\begin{array}{l}
x_{+}(0)=\bar{x}+h, \\
x_{+}(t) \in[\bar{x}+h, \bar{x}(\bar{t})] \quad \forall t \in\left[0, \tau_{+}\right], \\
x_{+}(t)=\bar{x}\left(t+\bar{t}-\tau_{+}\right) \quad \forall t \in\left[\tau_{+}, u(\bar{x})+\tau_{+}-\bar{t}\right] .
\end{array}\right.
$$

Notice that $x_{+}\left(u(\bar{x})+\tau_{+}-\bar{t}\right)=\bar{x}(u(\bar{x})) \in \mathbb{R}^{N} \backslash \Omega$, so that

$$
u(\bar{x}+h) \leq u(\bar{x})+\tau_{+}-\bar{t} .
$$

Proof of Step 2. In order to construct the line segment part, let us set $q_{+}=$ $\bar{x}(\bar{t})-(\bar{x}+h)$ and observe that, in view of $(45)$,

$$
q_{+}=\bar{t} \bar{v}+O\left(\bar{t}^{1+\alpha / 2}\right)-h .
$$

Then, $q_{+} \neq 0$ since $\bar{t}>>|h|$ and $|\bar{v}| \geq 1 / r$. Let $\lambda(\cdot)$ be a solution of the Cauchy problem

$$
\left\{\begin{array}{l}
\lambda^{\prime}(t)=\frac{1}{H^{0}\left(\bar{x}+h+\lambda(t) q_{+}, q_{+}\right)}, t \geq 0 \\
\lambda(0)=0 .
\end{array}\right.
$$

Since $\lambda(\cdot)$ is strictly increasing there is a unique time $\tau_{+}$such that $\lambda\left(\tau_{+}\right)=1$. Now, set

$$
x_{+}(t)=\bar{x}+h+\lambda(t) q_{+} \quad t \in\left[0, \tau_{+}\right] .
$$

Then $x_{+}(\cdot)$ is a solution of the differential inclusion $(28)$ on $\left[0, \tau_{+}\right]$because

$$
H^{0}\left(x_{+}(t), x_{+}^{\prime}(t)\right)=1 \quad \forall t \in\left[0, \tau_{+}\right] .
$$

Moreover $x_{+}\left(\tau_{+}\right)=\bar{x}(\bar{t})$. Thus, defining

$$
x_{+}(t)=\bar{x}\left(t+\bar{t}-\tau_{+}\right) \quad \forall t \in\left[\tau_{+}, u(\bar{x})+\tau_{+}-\bar{t}\right]
$$

completes the construction of $x_{+}(\cdot)$.

Step 3. We will now prove the estimate

$$
\tau_{+} \leq \bar{t}+\left\langle D_{q} H^{0}(\bar{x}, \bar{t} \bar{v}), q_{+}-\bar{t} \bar{v}\right\rangle+C \frac{|h|^{2}}{\bar{t}} .
$$

Proof of Step 3 To begin with, let us note that any $\xi \in\left[\bar{t} \bar{v}, q_{+}\right]$satisfies $|\xi| \geq$ $\bar{t} / C$. Indeed, if $\xi=\mu q_{+}+(1-\mu) \bar{t} \bar{v}$ for some $\mu \in[0,1]$, then, by (47), $\xi=$ 
$\bar{t} \bar{v}+O\left(\bar{t}^{1+\alpha / 2}\right)-h$ with $|h|=\bar{t}^{1 / \beta}$ and $\beta \in(0,1)$. So, for $|h|$ small enough, we have the desired claim: $|\xi| \geq \bar{t} / C$. Then, by Lemma 3.9 we conclude that the $\operatorname{map} \xi \mapsto D_{q} H^{0}(\bar{x}, \xi)$ is Lipschitz continuous on $\left[\bar{t} \bar{v}, q_{+}\right]$with constant $C / \bar{t}$. So, for all $t \in\left[0, \tau_{+}\right]$,

$$
\begin{aligned}
& H^{0}\left(x_{+}(t), q_{+}\right) \leq H^{0}\left(\bar{x}, q_{+}\right)+C\left|x_{+}(t)-\bar{x}\right|^{2 \alpha}\left|q_{+}\right| \leq H^{0}(\bar{x}, \bar{t} \bar{v}) \\
& \quad+\left\langle D_{q} H^{0}(\bar{x}, \bar{t} \bar{v}), q_{+}-\bar{t} \bar{v}\right\rangle+(C / \bar{t})\left|q_{+}-\bar{t} \bar{v}\right|^{2}+C\left|x_{+}(t)-\bar{x}\right|^{2 \alpha}\left|q_{+}\right| .
\end{aligned}
$$

Since $x_{+}(t) \in[\bar{x}+h, \bar{x}(\bar{t})]$, we have

$$
\left|x_{+}(t)-\bar{x}\right| \leq \max \{|h|,|\bar{x}(\bar{t})-\bar{x}|\} \leq C \bar{t} .
$$

Also, on account of (47) and (43),

$$
\left|q_{+}\right| \leq C \bar{t} \quad \text { and } \quad\left|q_{+}-\bar{t} \bar{v}\right|=\left|O\left(\bar{t}^{1+\alpha / 2}\right)-h\right| \leq C|h| .
$$

Noting that $H^{0}(\bar{x}, \bar{v})=1$ because $\bar{v} \in \partial F(\bar{x})$, the above inequality yields, by the homogeneity of $H^{0}(\bar{x}, \cdot)$,

$$
H^{0}\left(x_{+}(t), q_{+}\right) \leq \bar{t}+\left\langle D_{q} H^{0}(\bar{x}, \bar{t} \bar{v}), q_{+}-\bar{t} \bar{v}\right\rangle+C\left(\frac{|h|^{2}}{\bar{t}}+\bar{t}^{1+2 \alpha}\right),
$$

where $|h|^{2} / \bar{t}>\bar{t}^{1+2 \alpha}$ because $\bar{t}=|h|^{\beta}$ with $\beta>1 /(1+\alpha / 2)$. So,

$$
H^{0}\left(x_{+}(t), q_{+}\right) \leq \bar{t}+\left\langle D_{q} H^{0}(\bar{x}, \bar{t} \bar{v}), q_{+}-\bar{t} \bar{v}\right\rangle+C|h|^{2} / \bar{t} .
$$

Then

$$
1=\int_{0}^{\tau_{+}} \lambda^{\prime}(t) d t=\int_{0}^{\tau_{+}} \frac{d t}{H^{0}\left(x_{+}(t), q_{+}\right)} \geq \frac{\tau_{+}}{\bar{t}+\left\langle D_{q} H^{0}(\bar{x}, \bar{t} \bar{v}), q_{+}-\bar{t} \bar{v}\right\rangle+C|h|^{2} / \bar{t}},
$$

which in turn yields (48).

Conclusion. Repeating the above reasoning with $q_{-}=\bar{x}(\bar{t})-(\bar{x}-h)$, we can build a solution $x_{-}(\cdot)$ to $(28)$ such that $x_{-}(0)=\bar{x}-h, x_{-}(t) \in[\bar{x}-h, \bar{x}(\bar{t})]$ on the time interval $\left[0, \tau_{-}\right]$, and $x_{-}(t)=\bar{x}\left(t+\bar{t}-\tau_{-}\right)$on $\left[\tau_{-}, u(\bar{x})+\tau_{-}-\bar{t}\right]$. Therefore,

$$
u(\bar{x}-h) \leq u(\bar{x})+\tau_{-}-\bar{t},
$$

where $\tau_{-}$can be estimated as above:

$$
\tau_{-} \leq \bar{t}+\left\langle D_{q} H^{0}(\bar{x}, \bar{t} \bar{v}), q_{-}-\bar{t} \bar{v}\right\rangle+C \frac{|h|^{2}}{\bar{t}} .
$$

Hence, by (46), (49), (48), (50), and (45) we obtain

$$
\begin{aligned}
u(\bar{x}+h)+u(\bar{x}-h)-2 u(\bar{x}) & \leq \tau_{+}-\bar{t}+\tau_{-}-\bar{t} \\
& \leq 2\left\langle D H^{0}(\bar{x}, \bar{t} \bar{v}), \bar{x}(\bar{t})-\bar{x}-\bar{t} \bar{v}\right\rangle+C \frac{|h|^{2}}{\bar{t}} \\
& \leq C|h|^{\beta(2+\alpha) / 2}
\end{aligned}
$$

since $\beta<4 /(4+\alpha)$. We have thus attained (44), which completes the proof. 


\section{Acknowledgments}

The authors thank the referee for bringing reference [8] to their attention. This work was partially supported by the Commission of the European Communities under the 7th Framework Programme Marie Curie Initial Training Network (FP7-PEOPLE-2010-ITN), project SADCO, contract number 264735 .

\section{References}

[1] Bardi, M., Capuzzo Dolcetta, I.: Optimal Control and Viscosity Solutions of Hamilton-Jacobi-Bellman Equations. Springer, Berlin (1997)

[2] Cannarsa, P., Frankowska, H.: Some characterizations of optimal trajectories in control theory. SIAM J. Control Optim. 29, 1322-1347 (1991)

[3] Cannarsa, P., Sinestrari, C.: Semiconcave Functions, Hamilton-Jacobi Equations and Optimal Control. Birkhäuser, Boston (2004)

[4] Cannarsa, P., Soner, H.M.: Generalized one-sided estimates for solutions of Hamilton-Jacobi equations and applications. Nonlinear Anal. 13(3), 305-323 (1989)

[5] Cardaliaguet, P., Ley, O., Monteillet, A.: Viscosity solutions for a polymer crystal growth model. Indiana Univ. Math. J. (to appear)

[6] Ishii, H.: Uniqueness of unbounded viscosity solutions of Hamilton-Jacobi equations. Indiana Univ. Math. J. 33, 721-748 (1984)

[7] Lions, P.L.: Generalized Solutions of Hamilton-Jacobi Equations. Pitman, London (1982)

[8] Lytchak, A., Yaman, A.: On Hölder continuous Riemannian and Finsler metrics. Trans. Am. Math. Soc. 358(7), 2917-2926 (2006)

[9] Sinestrari, C.: Semiconcavity of solutions of stationary Hamilton-Jacobi equations. Nonlinear Anal. 24, 1321-1326 (1995)

[10] Su, B., Burger, M.: Global weak solutions of non-isothermal front propagation problem. Electron. Res. Announc. Am. Math. Soc. 13, 46-52 (2007)

Piermarco Cannarsa

Dipartimento di Matematica

Università di Roma "Tor Vergata"

Via della Ricerca Scientifica 1

00133 Rome

Italy

e-mail: cannarsa@mat.uniroma2.it 
Pierre Cardaliaguet

CEREMADE

Université Paris-Dauphine

UMR CNRS 7534

Place du Maréchal De Lattre

De Tassigny

75775 Paris Cedex 16

France

e-mail: cardaliaguet@ceremade.dauphine.fr

Received: 15 March 2011.

Accepted: 18 December 2011. 